\title{
Applications and Challenges of Cloud Computing and check its status in Iran
}

\author{
Yaghub POURASAD ${ }^{1}$, Zahra Rajab LARIJANI ${ }^{2}$, Zabihollah JISTAN ${ }^{3}$ \\ ${ }^{1}$ Faculty of Electrical, University of Urmia, West Azarbaijan, Iran \\ ${ }^{2}$ Computer Department, Faculty of Engineering, Tehran Branch, Islamic Azad University, Tehran, Iran \\ ${ }^{3}$ Computer Department, Faculty of Engineering, Tehran Branch, Islamic Azad University, Tehran, Iran
}

\begin{abstract}
Nowadays the administrative system tailored to developing communications and the emergence of various streams of communication, has been further extended to the extent that the key to organizational survival and the continuation of their activities, is armed with these organizations means new competition, the diverse systems and applications in various areas of information technology. A new model that we can provide computing resources and the services provided through the Internet have called cloud computing. Using cloud computing power efficiency and saving it resources and computing power increases. Cloud computing is of the opinion that Internet-based resources, faster and with lower costs and more variety can be used. Take advantage of network-based cloud computing demand large amounts of computational resources with lower costs and provides access from any location and at any time with an internet connection. In this paper, at first we propose a definition and general concepts of cloud computing and continue to explore the advantages and disadvantages and challenges to consider the assessment of the state of cloud computing will be in Iran.
\end{abstract}

Keywords: cloud computing, cloud models, challenges cloud, cloud security

\section{Introduction}

Cloud computing by computer scientist John McCarthy in 1960 is predicted to be a new term, but as a concept has entered the field of technology. McCarthy said: "computation may someday be organized as a public industry." John McCarthy foresight to predict the cloud computing concept that we know today.

Cloud computing since 2007 and are the most important issues of commercial and scientific research. Cloud computing based on shared computing resources to act. In this service, customers can pay for computing resources

The world of computing is moving quickly to develop software that runs on computers rather than individual, as a service available to millions of consumers are put. [1] From this point of view, cloud computing (Cloud Computing) from the perspective of end users is structurally similar to a cloud 
through which they can access applications from anywhere in the world. But cloud computing infrastructure from the perspective of resource providers, can help with virtual machine network, as a new method for creating dynamic new generation of data centers used to provide a flexible infrastructure to provide different types of computing services have the storage.

\section{The concept and nature of cloud computing}

Cloud computing is a model for providing information technology services by means of the Internet and web-based applications rather than direct connection to a server, are received. Data is stored on a server and software packages. However, cloud computing access to information structure as much as the electronic devices are allowed access to the Internet. Take. Another cloud computing model to calculate a new look in 2006, as well as the evolutionary origins of parallel computing, distributed computing, grid computing estimates and raising the efficiency of network capacity, virtualization and load balancing (such as split processing between available resources) is considered. [1]

All services in cloud computing / cloud resources and cloud management provider. Cloud computing makes its customers with a lower cost of access to shared resources .The basic principle of cloud computing is that users' data is not stored locally but are stored in the data center. The important benefits of cloud computing for developers has created,is The possibility of setting up cloud masses in places of the world That the cost of room and power consumption are more affordable. This will provide greater security against natural disasters that may occur in a certain area and Provide possible traffic and load adjustment system to perform queries in clusters that are physically closer to the user.[2]

Cloud computing provides applications of storage, compute and use of the Internet for users. For web developers a software development platform and runtime environment is an Internet-scale software And for infrastructure managers and providers of communication, such as a massive distributed data centers is based infrastructure that are connected through the network.[3]

\section{Components and features cloud-computing model}

Some companies such as NIST company presented five features for cloud computing:

\section{Elasticity with speed}

Be possible to quickly and flexibly, in some cases automatically, to quickly expand been awarded (in view of the scale) or be released on the spot and quickly achieve a smaller scale. The sight of the customer to use the possibilities that are available often seem unlimited and can be purchased in any amount and at any time.

\section{Car service on demand}

May be new or increased consumer demand computing capacity they have. The increase automatically without any human interaction done on the part of the service provider. Amazon Web Service is an example of it. 


\section{Calculated Services}

Cloud systems automatically control and optimize their resources. This ability is measured by applying the appropriate level of service or supply is used (eg: storage, processing power, bandwidth and the number of active users) is performed. The use of resources for both the provider and the customer can be clearly monitored, controlled and reported.[4]

\section{Analysis of Sources}

Assemble resources to deliver optimized computing services can model the "many customers" to serve multiple users. This is different from the real and virtual resources dynamically based on customer requests and then getting them done. For example, resources include storage, processing power, memory, network bandwidth, and virtual machines.

\section{Achieving wide area network (WAN)}

Access to an extensive network means that cloud services can be accessed from any platform. Internet as its backbone and can be standard mechanisms to achieve them. This mechanism of diverse media (mobile phones, laptops and PDA's) will support

\subsection{This service models}

Saas : This model, the usability of the applications running on the cloud provides to consumers. Applications of devices of different customers, through a web browser interface are available. No need to install and quick and easy to define new users and pay-per-use model, SaaS services into a great opportunity for individuals and organizations has become.

In this model, consumers can not control or management on basic infrastructure cloud, even individual application capabilities, with the possible exception configuration settings for specific applications are limited. Google Translate, Sasleforce, Oracle are examples of this model.[5]

Pass: This service model, computing resources, applications and services with a platform that can be built and deployed it provides. Services PaaS, online platforms to create, test and deploy Web applications and can provide them with the use of browser-based software development tools and building, used. Build a program using PaaS services than the traditional approach in programming and software development, with less effort and work done in a shorter time in addition, install and configure software development platforms and tools and programs is not required.[6]

In this model, consumers can control over the deployed applications and possibly application hosting environment configurations have settings. Google App Engine, Microsoft Azure, Force.com are examples of this model.

Iaas: Infrastructure as a service that allows consumers to different types of infrastructure, including processing, storage, networks and other fundamental computing resources, achieve and your favorite software, including operating systems or applications are deployed and run. Consumers can not control the physical hardware, but they can be on operating systems, storage, applications, and possibly limited selection of network components such as host firewalls, to 
have control. In fact IaaS hardware without hassle, the cost of purchasing and managing the hardware offers.[6]

Because all are virtualization-based cloud infrastructure, server software can operate independently of the hardware infrastructure and multiple virtual machines (operating systems and storage software applications installed) can run on only one computer and its processing power PC to be used jointly. This enables cloud service providers to storage media, computing and software resources to be managed simultaneously, and therefore the demand to consumers. Rackspace, Amazon S3, Windows Azure Sql, Mosso Akami, are examples of infrastructure service providers.

Daas: Database as a service an attractive interface to access and organize data creates. The advantage is ease of use in servers, software or special hardware is required. Manage it not the responsibility of the user and the database administrator does not need.[7]

Naas: Network as a service that uses the cloud provider to offer customers a virtual network and provides services for the transport network.[7]

\section{2. offer models and expand of this service}

\section{Public cloud}

This model is clearly seen. Amazon Web, Google App engine, Microsoft Azure are examples of public cloud.

\section{Private Cloud}

The cloud infrastructure is made for private organizations. This may be administered by an organization or a third party and on premise or off premise may also be.

In this model, customized cloud services, are deployed within organizations, so the data only for internal users is the ability to achieve. This approach for organizations that focus on data security and privatization or to facilitate or to change the way people work, is appropriate. In other words, many large organizations, rather than the server, software and data in their data centers and private clouds are providing this opportunity for them. This cloud use software, such as VM Ware, VClouds Director, etc., that enable the use of cloud capabilities. Most users who use public cloud services must pay a monthly fee instead, services and facilities that receive as previously mentioned because of their cloud nature to use this feature the user dose not need prepare or upgrade hardware and software using this service is very affordable and if you need more features Immediately supply the needs of the user. The use of these services is very diverse For example, you can save important files with very large amounts of data by this service. As mentioned above a monthly fee should be paid to use this service so additional costs must be paid if you want your data to be stored longer in the service. Often, public cloud service is used for dynamic data

\section{Group cloud}

Organizations wich have common goals implement this cloud together. Computing resources for organizations that share common goals provided by Internet. The cloud is managed by the organization or the other person. The cloud infrastructure can exists in an special organization or all across organizations. 


\section{Hybrid cloud}

The cloud is a combination of public,private and forumsclouds computing resources. Its advantage is its flexibility as in some cases the private or public cloud resources can be changed. For example, increases in workload, Some calculations can be carried out either in the public cloud or private cloud.[8]

\section{Advantages and Disadvantages of Cloud Computing}

\subsection{Advantages of cloud computing}

Availability: It can be anywhere and any time (with Internet connection) to access information. Power outages, fires and natural disasters, can serve the organization for hours and perhaps days disrupt while the service providers known as the Disaster Recovery Plan has plans to return to the normal situation as soon as possible.[9]

Maximum efficiency from the server: The use of cloud computing is guaranteed to allocate resources in the best possible manner among all users upgrade.

Backup: Since in most cases the provider's backup in case of any problems, you can access a copy of the information.

Interoperability: The resources can be used for a common goal.

Green technology: Optimal use of resources makes energy saving.

reduction in costs: Users are not paying for new sources. In most cases, users can connect to the Internet using a browser to access resources and services they need. It is alleged that the technology greatly reduces costs. Cloud save client from hardware, software and services costs also the installation and maintenance of business applications is locally absent.

Extensibility: Prediction hardware and software requirements when planning the budget in many cases will be wrong while in public cloud services in a very short time, can be increased or decreased. For example, If the organizations web client increase due to unexpected reason you can ask the service provider to increase resources dedicated to website.[9]

Improving Performance: Faster and more accurate data retrieval is performed.

Security: According to centralize the data and the use of security resources more and more complex and the presence of certified security professionals can increase security, but there are many concerns.

\subsection{Disadvantages of using cloud computing}

Security problems: Data theft, data loss, theft and traffic service account, API unsafe, attacks Denial of Servic, fellow traitor, misuse of the Service Cloud, Shared Technology Vulnerabilities. 
Bulletin de la Société Royale des Sciences de Liège, Vol. 86, special edition, 2017, p. 484 - 493

\section{Storage limits}

The problem in low-speed connection: Send and receive data at high volumes or documents may take many time.

Lack of control: your data in the cloud can be managed and controlled, but have no control over others information and services.

Bandwidth problems: Cloud providers need high bandwidth to provide appropriate services to a large number of the user.

Lack of transparency: public cloud users are not aware of the details of the implementation of cloud and data storage.

Being dependent on the service provider cloud: If the cloud provider for any reason have not fulfilled their obligations and not to other services, to the amount of information stored in the cloud .Problems encountered by users.

\section{The decline of cloud computing}

\subsection{Security and Privacy}

Its the biggest challenge of cloud computing. Concerns such as data integrity, data access, confidentiality, privacy and so on. There is little control over the data on the Internet. Security threats can be internal or external. On the other provider can access information and change or delete information intentionally or unintentionally. For example, hackers can use the cloud as an infrastructure with a cheaper price(For Myryt bat net ). Cloud service providers to meet and to minimize the concerns Uses data encryption, Allowed user authentication, accepted standards and service level agreements. privacy and security issues, can be encrypted using procedures, hardware and software security to some extent be resolved.

\section{Security in cloud computing is divided into six sub-categories}

1- Mechanisms for monitoring cloud servers 2- provide privacy for sensitive data 3- prevent illegal access provider staff 4- prevent data theft and phishing attacks and fraud 5 -security in multi-tenant virtual environments. 6- develop legislation for cloud users and providers[10]

\subsection{Interoperability and Portability}

In order cooperation between the clouds, the software on one platform, should be able to combine different services platform, or on the run. This is possible through web services, but writing these web services is complex. Another challenge of cloud computing is that the applications, platforms and data should be easily transferred from one cloud provider to another. So far this problem has not been solved because each cloud providers, several standard languages are used for their platform and the lack of rules and protocols for cooperation between the clouds felt. 


\subsection{Performance and Functionality Cloud}

Cloud performance is measured with the ability to run applications in the cloud. The lack of appropriate resources such as disk space, bandwidth, low cpu speed communication network, the performance will be affected and is low. Low efficiency makes the missing customers and provide cloud services to end.

\subsection{Ensure availability of the service}

Because of Frequent interruptions communication infrastructure and the Internet and internal technical problems cloud service providers still provide a stable cloud services for sensitive businesses have many problems Not very good quality, speed limit and inadequate coverage factor of the Internet are another problem in the cloud. Develop programs to monitor usage, SLA's, performance, capabilities and business affiliation to provide the client is crucial. One of the ways to gain the confidence is attached resource use.

\section{5. points of weakness}

Cloud access via the Internet it is possible that this could cause a problem. The problem with the Internet in any way will not have access to the cloud. If you use a cloud service provider, by creating problems for servers will not have access to cloud services.

\section{6. scheduling and resource allocation}

In the cloud computing approach for the resources they demand a certain sum paid and the resources placed at the disposal for a specified period. The drawbacks of this method is that the high level of system workload, a work by a given customer demand have much time to wait until the reduced workload The work to be addressed.

The current allocation methods such as FIFO, Roun-Robin, etc. that are used in the Clouds doing unfair allocation regardless of the priority tasks. Scheduling tasks is one of the critical calculations. A wrong choice in the scheduling calculations can log in to cause adverse effects on performance. Using virtualization technology, using Round Robin scheduling algorithm, scheduling algorithm working life of bees, etc. can be used as a solution to this challenge.

\section{7. transparency}

Who has access to user data and does not prevent users from accessing unauthorized data, is yet another challenge. In the public cloud customer does not provide any information and access to data centers services. As a solution to these challenges, organizations have to monitor third service provider systems and provider practices designed to respond to clients' document. [10]

\subsection{Virtualization}

Virtualization is a virtual version of everything (storage devices, operating systems, servers or network resources) will be produced. This will turn resources into multiple execution environment that hide their physical characteristics. This reduced hardware and energy costs. The benefits of virtualization are, flexibility and scalability, reduce costs, personalization and 
user interface with easy access. However, the virtualization make infrastructure management more difficult.

\section{9. bandwidth costs}

That companies using cloud can save the hardware costs and data center designs and also the cost of skilled labor is an sides of the issue. Another issue is the high cost of additional bandwidth available to cover the needs of cloud services and The availability of Enterprise Cloud. The cost is minimal for small applications but for systems with large amounts of data and processing is involved, is very high.[11]

\section{Cloud Computing and the situation in Iran}

Discuss cloud computing (cloud computing) not long history in Iran, Now, however, Iran does not open the technology in place and users prefer to store data on memory cards and away from cyberspace, but global corporations put such services to users industrialized countries. Of course, users prefer this service.

But this functional system, has not yet penetrated in Iran; goes back to lack of awareness and lack of confidence and limited access to inappropriate Internet bandwidth.

Cloud computing is discussed in more specialized meetings university raised in Iran And still did not reach the macro-level policies. The guild system has been computerized, including parts to enter the cloud computing and the need for it in Iran has maintained and enhanced.

In a report released by the Society of cloud computing and cloud computing Amirkabir University of Technology in collaboration with the Research Center published in 93 years, the results are as follows.

Participants with at least a bachelor's degree and were more than a year of cloud computing services. Despite the varied benefits of cloud computing adoption, as well as the problems: Cloud computing inefficiency in the absence of sufficient bandwidth, lack of resources needed for the use of cloud computing, lack of adequate security in the use of cloud computing services. Some of the characteristics of cloud computing is also ambiguous in its adoption, including: In compliance with the norms ambiguity, uncertainty in the difference or similarity with previous experience, the ambiguity of how integration with existing infrastructure, uncertainty in how to manage time, doubt the existence of the necessary support, security ambiguity, uncertainty in financial management.

The results of this study indicate the capacity of the country's top adoption of cloud computing the provision of appropriate infrastructure and resources and appropriate support, a lot of benefits to be realized within the user in short, the flexibility in IT that can increase business efficiency and better management of time and costs.[12 


\section{Conclusion}

With regard to the issues raised can be concluded that the topic of cloud computing is inherently useful and beneficial. But on the condition that its use culture to be considered and proper education for its users. Because according to the security problem that may use this technology with knowledge inadequate or lax irreparable damages import. However, due to the large companies in the technology, perhaps in near future we all have a dedicated Electronic cloud. Despite the expansion of cloud computing, there are many advantages and disadvantages, but the technology is still in its infancy. Relying on its many benefits, the interest in using cloud services is growing. To meet the challenges and problems like security and privacy, etc. acceptance and use of its bandwidth increases. To meet the challenges and problems like security and privacy, etc. acceptance and use of its bandwidth increases. This paper examines the advantages and disadvantages of cloud computing models examined and discussed some of the challenges it. With the widespread use and acceptance of cloud and ideas and universal access to the Internet without boundaries through the clouds, big companies build smart devices, remove the old storage systems pose. If implementation of the plan, the countries that are lagging behind cloud storage, will be in big trouble.

\section{References}

[1] Rajkumar Buyya, Chee Shin Yeo, Srikumar Venugopal, James Broberg, and Ivona Brandic, "Computing as the 5th Utility," Future Generation Computer Systems, Volume 25, Number 6, PP Cloud Computing and Emerging IT Platforms: Vision, Hype, and Reality for Delivering. 599-616, ISSN: 0167-739X, Elsevier Science, Amsterdam, The Netherlands, June 2009.

[2] Aymerich, F.M., Fenu, G., Surcis, S., "An approach to a Cloud Computing network," ICADIWT 2008. First International Conference on Applications of Digital Information and Web Technologies, 4-6 Aug. 2008. PP.113 - 118

[3] Mojtaba Jalali, Mojtaba Rezvani, Maryam Tajobyan, Cloud Computing: Implications, Challenges and Applications, Second National Conference on Emerging Trends in Computer Engineering Young Researchers and Elite Club

[4] Khosravi, b. 1390. Introduction to cloud computing and its application in the organization. First Edition. Isfahan emissions Touba nesf e jahan.

[5] Bhardwaj S, Jain L, Jain S. 2010." An Approach for Investigating Perspective of Cloud Software-as-a-Service (SaaS)". International Journal of Computer Applications, 40-43p

[6] Lin, Geng, Fu, David, Zhu, Jinzy, Dasmalchi, Glenn, "Cloud Computing: IT as a Service," IT Professional, Volume 11, Issue 2, March-April 2009. PP.10 - 13

[7] Abolhasani Jamal, Rostaee Rasool, Zohre vandi Ebadollah, cloud computing, applications and challenges, the second National Conference on Emerging Trends in Computer Engineering Young Researchers and Elite Club

[8] Tharam Dillon and Chen Wu and Elizabeth Chang، "Cloud Computing : Issues and Challenges" "University of Technology Perth, Australia ،24th IEEE ،2010 .

[9] Ishita Verma," Cloud Computing: A study of Benefits and Challenges" IJASCSE,Volume 3, Issue 7, 2014.

[10] Sanjita Das, Ankita Chandrakar, Reshamlal Pradhan ," A Review On Issues And Challenges Of Cloud Computing” IJIACS, Volume 4, Issue 1 January 2015. 
Bulletin de la Société Royale des Sciences de Liège, Vol. 86, special edition, 2017, p. 484 - 493

[11] Abílio Cardoso and Paulo Simões," Cloud Computing: Concepts, Technologies and Challenges" CCIS 248, pp. 127-136, 2012

[12] Cloud Computing Research Center Amirkabir University of Technology( AUT): http://crc.aut.ac.ir 